\author{
S.Sh. Kazhikenova ${ }^{1}$, M.I. Ramazanov ${ }^{2}$, A.A. Khairkulova ${ }^{1}$, G.S. Shaikhova ${ }^{1}$ \\ ${ }^{1}$ Karaganda State Technical University, Kazakhstan; \\ ${ }^{2}$ Ye.A. Buketov Karaganda State University, Kazakhstan \\ (E-mail: aitbekovna3@mail.ru)
}

\title{
$\varepsilon$-Approximation of the temperatures model of inhomogeneous melts with allowance for energy dissipation
}

\begin{abstract}
Accumulated facts and information about the Navier-Stokes equations, together with a large number of experiments and approximate calculations, made it possible to reveal some discrepancies between the mathematical model of a viscous melt and real phenomena in the nature of real molten systems. There are many reasons for this. One of them is the nonlinearity of the Navier-Stokes equations. And for nonlinear equations it is known that in non-stationary problems, a solution satisfying it can exist not on the entire interval $t \geq 0$. Over a finite period of time, it can either go to infinity, or crumble.A solution lose regularity and no satisfying the equations and begin branching. It is mathematically proved that if this solution exists for $t \geq 0$, then it may not seek to solve the stationary problem when stabilizing the boundary conditions and external influences. The solutions of the nonstationary problem obtained even with a smooth initial regime and smooth external influences can become less regular with time, and then generally go into irregular or turbulent regimes. The actual implementation of this or that branch of the solution depends on extraneous reasons not taken into account in the Navier-Stokes equations. In the proposed paper, we constructed a numerical scheme with good convergence. The regularization of the initial systems of differential equations by $\varepsilon$-approximation is constructed. The Galerkin method is implemented ensuring the correctness of boundary value problems for an incompressible viscous flow both numerically and analytically. A splitting scheme for the Navier-Stokes equations with a weak approximation is constructed. An approximation is constructed for stationary and nonstationary models of an incompressible melt, which leads to nonlinear equations of hydrodynamics to a system of equations of Cauchy-Kovalevskaya type.
\end{abstract}

Keywords: energy dissipation, system approximation, Cauchy inequality, Galerkin method, a priori estimates.

It is known that the Navier-Stokes equations are analytically insoluble, and if they are solvable, then in relatively simple cases.

To solve the Navier-Stokes equations, a nonconformal finite element method was proposed in [1]. It is based on the modification of the Navier-Stokes equations with the introduction of weight functions. The solution of the Navier-Stokes equations with respect to natural variables, from which pressure is excluded, is considered in [2]. This approach is already known. Thus, the Navier-Stokes equations system is integrated numerically using a finite-difference method using splines. Other methods are also being sought. An attempt is made to derive the Navier-Stokes equation itself using the variational method, as presented in [3].

We investigate the initial-boundary value problem for nonstationary Navier-Stokes equations in this section. Let us consider the temperature model of an inhomogeneous melt [4] in the region $\Omega \subset R^{2}$.

$$
\begin{gathered}
\rho\left(\frac{\partial v}{\partial t}+(v \cdot \nabla) v\right)=\mu \Delta v-\nabla p+e \theta \rho+\rho f ; \\
\frac{\partial \rho}{\partial t}+(v \cdot \nabla) \rho=0 \\
\operatorname{div} v=0 \\
\rho\left(\frac{\partial \theta}{\partial t}+(v \cdot \nabla) \theta\right)=\operatorname{div}(\lambda(\theta) \nabla \theta)+\mu \sigma ; \\
\sigma=\sum_{i, j=1}^{2}\left(\frac{\partial v_{i}}{\partial x_{j}}+\frac{\partial v_{j}}{\partial x_{i}}\right)^{2}
\end{gathered}
$$


with initial-boundary conditions

$$
\begin{gathered}
\left.v\right|_{t=0}=v_{0}(x),\left.\rho\right|_{t=0}=\rho_{0}(x),\left.\theta\right|_{t=0}=\theta_{0}(x) ; \\
\left.v\right|_{S}=0,\left.\frac{\partial \theta}{\partial n}\right|_{S}=0, t \in[0, T],
\end{gathered}
$$

where $\sigma$ - energy dissipation; $v(x, t)$ - velocity vector; $\theta(x, t)$ - temperature field; $\rho(x, t)$ - density field; $p(x, t)$ - pressure field; $f(x, t)$ - mass force vecto; $\mu$ - melt viscosity; $\lambda(\theta)$ - thermal conductivity coefficient; $n$ is the outer normal to the boundary $S, e=\{0,1\}$.

Solvability of the problem (1)-(6) was studied in Sh.S. Smagulova and A.B. Kazhikhova $[5,6]$.

The system of equations (1)-(5) is non-evolutionary, therefore direct application of the method of fractional steps is difficult. In connection with this we study the approximation of the system (1)-(5) by a system of evolutionary type and study the existence theorem for the solution of the auxiliary problem. Let the motion of the melt occur in a bounded region $\Omega \subset R^{2}$ with a sufficiently smooth boundary $S$. For simplicity we assume that the boundary $S$ is impermeable and there is no mass transfer between the melt and the external medium.

We consider a system of equations with a small parameter approximating the system of equations (1)-(5):

$$
\begin{gathered}
\rho^{\varepsilon}\left(\frac{\partial v^{\varepsilon}}{\partial t}+\left(v^{\varepsilon} \cdot \nabla\right) v^{\varepsilon}\right)=\mu \Delta v^{\varepsilon}-\nabla p^{\varepsilon}+e \theta^{\varepsilon} \rho^{\varepsilon}+\rho^{\varepsilon} f-\rho^{\varepsilon} \frac{v^{\varepsilon}}{2} d i v v^{\varepsilon} \\
\frac{\partial \rho^{\varepsilon}}{\partial t}+\left(v^{\varepsilon} \cdot \nabla\right) \rho^{\varepsilon}=0 \\
\varepsilon p^{\varepsilon}+d i v v^{\varepsilon}=0 \\
\rho^{\varepsilon}\left(\frac{\partial \theta^{\varepsilon}}{\partial t}+\left(v^{\varepsilon} \cdot \nabla\right) \theta^{\varepsilon}\right)=\operatorname{div}\left(\lambda\left(\theta^{\varepsilon}\right) \nabla^{\varepsilon} \theta^{\varepsilon}\right)+\mu \sigma^{\varepsilon} \\
\sigma^{\varepsilon}=\sum_{i, j=1}^{2}\left(\frac{\partial v_{i}^{\varepsilon}}{\partial x_{j}}+\frac{\partial v_{j}^{\varepsilon}}{\partial x_{i}}\right)^{2}
\end{gathered}
$$

with initial-boundary conditions:

$$
\begin{gathered}
\left.v^{\varepsilon}\right|_{t=0}=v_{0}(x),\left.\rho^{\varepsilon}\right|_{t=0}=\rho_{0}(x),\left.\theta^{\varepsilon}\right|_{t=0}=\theta_{0}(x) ; \\
\left.v^{\varepsilon}\right|_{S}=0,\left.\frac{\partial \theta^{\varepsilon}}{\partial n}\right|_{S}=0, t \in[0, T],
\end{gathered}
$$

Before proceeding to the proof of the theorem, we formulate an important definition.

Definition.A function $(v, p, \rho, \theta)$, that is summable together with the derivatives entering into the system of equations (1)-(6) satisfying (1)-(5) almost everywhere in the corresponding measure is called a strong solution of the problem (1)-(6).

The definition of the strong solution of the problem (7)-(12) is defined similarly.

Theorem 1. Let $f \in L_{p}(Q), \Omega \subset E^{2}, v_{0}(x) \in W_{p}^{1}(\Omega), 0<m \leq \rho_{0}(x) \leq M<\infty, \lambda(\theta)$ - is continuously differentiable with respect to

$\theta, \rho_{0}(x) \in W_{p}^{1}(\Omega), p>2, \lambda(\theta) \sim \theta^{2}$, at $\theta \rightarrow \infty, \theta_{0}(x) \in L_{\infty}(\Omega), \theta_{0} \in L_{p}(\Omega), \varepsilon>0, S \in C^{2}, \mu>0$.

Then a unique strong solution of problem (7)-(12) exists and we have the estimate for the solution, where $\mathrm{C}$ - the constant does not depend on

$$
\left\|\frac{\partial v^{\varepsilon}}{\partial t}\right\|_{L_{p}\left(0, T, L_{p}(\Omega)\right)}+\left\|v^{\varepsilon}\right\|_{L_{p}\left(0, T, W_{p}^{2}(\Omega)\right)}+\frac{1}{\varepsilon}\left\|d i v v^{\varepsilon}\right\|_{L_{p}\left(0, T, L_{p}(\Omega)\right)}+\left\|\rho^{\varepsilon}\right\|_{W_{p}^{1,1}(Q)}+\left\|\theta^{\varepsilon}\right\|_{W_{p}^{2,1}(Q)} \leq C<\infty .
$$

The proof of the theorem is constructed from three stages: obtaining a priori estimates, applying Galerkin's method for constructing approximate solutions and limiting the transition.

A priori estimates.

We have by the maximum principle:

$$
0<m \leq \rho_{0}^{\varepsilon}(x) \leq M<\infty .
$$


We multiply equation (7) scalarly in $v^{\varepsilon}(x, t)$ space and integrate by parts. Applying the Cauchy inequality:

$$
\left|\int_{\Omega} \rho^{\varepsilon}\left(f, v^{\varepsilon}\right) d x\right| \leq\left(\int_{\Omega} \rho^{\varepsilon}\left|v^{\varepsilon}\right|^{2} d x\right)^{\frac{1}{2}}\left(\int_{\Omega} \rho^{\varepsilon}|f|^{2} d x\right)^{\frac{1}{2}} .
$$

We have the estimate on the basis of imbedding theorems:

$$
\left\|v_{x}^{\varepsilon}\right\|_{L_{p}\left(0, T, L_{p}(\Omega)\right)}+\frac{1}{\varepsilon}\left\|d i v v^{\varepsilon}\right\|_{L_{p}\left(0, T, L_{p}(\Omega)\right)} \leq C<\infty .
$$

We obtain further multiplying (10) by $\theta_{t}^{\varepsilon}$, integrating by parts $\Omega$ :

$$
\begin{gathered}
\frac{1}{2} \frac{d}{d t} \int_{\Omega} \lambda\left(\theta^{\varepsilon}\right) \theta_{x}^{\varepsilon^{2}} d x+\int_{\Omega} \rho^{\varepsilon} \theta_{t}^{\varepsilon^{2}} d x= \\
=\int_{\Omega} \frac{1}{2}\left\{\lambda^{\prime}\left(\theta^{\varepsilon}\right) \theta_{x}^{\varepsilon^{2}} \cdot \theta_{t}^{\varepsilon}\right\} d x+\int_{\Omega} \mu \sigma^{\varepsilon} \theta_{t}^{\varepsilon} d x-\int_{\Omega} \rho^{\varepsilon}\left(v^{\varepsilon} \cdot \nabla\right) \theta^{\varepsilon} \cdot \theta_{t}^{\varepsilon} d x .
\end{gathered}
$$

We estimate the integrals on the right-hand side and integrate with respect to the variable t:

$$
\max _{0 \leq t \leq T}\left\|\theta_{x}^{\varepsilon}\right\|_{2, \Omega}^{2}+\left\|\theta_{t}^{\varepsilon}\right\|_{2, Q}^{2} \leq C .
$$

We will write down the energy equation:

$$
\rho^{\varepsilon} \theta_{t}^{\varepsilon}-\left(\lambda\left(\theta^{\varepsilon}\right) \Delta \theta^{\varepsilon}\right)=\mu \sigma^{\varepsilon}-\rho^{\varepsilon}\left(v^{\varepsilon} \cdot \nabla\right) \theta^{\varepsilon}+\lambda^{\prime}\left(\theta^{\varepsilon}\right) \cdot \theta_{x}^{\varepsilon^{2}}
$$

and multiply it by $\frac{1}{\rho} \Delta \theta^{\varepsilon}$. We have After integration $\Omega$ :

$$
\frac{1}{2} \frac{d}{d t} \int_{\Omega} \theta_{x}^{\varepsilon^{2}} d x+\int_{\Omega} \lambda\left(\theta^{\varepsilon}\right) \frac{1}{\rho}\left(\Delta \theta^{\varepsilon}\right)^{2} d x=\int_{\Omega}\left\{\rho\left(v^{\varepsilon} \cdot \nabla\right) \theta^{\varepsilon}-\mu \sigma-\lambda^{\prime}\left(\theta^{\varepsilon}\right) \theta_{x}^{\varepsilon^{2}}\right\} \times \frac{1}{\rho} \Delta \theta^{\varepsilon} d x .
$$

We find after integrating over the variable $t$, estimating the integrals on the right-hand side:

We conclude as a result:

$$
\max _{0 \leq t \leq T}\left\|\theta_{x}^{\varepsilon}\right\|_{2, \Omega}^{2}+\left\|\Delta \theta_{t}^{\varepsilon}\right\|_{2, Q}^{2} \leq C
$$

$$
\left\|\theta^{\varepsilon}\right\|_{W_{p}^{2,1}(Q)} \leq C<\infty
$$

We obtain multiplying equation (8) by $\mathrm{u}$, integrating by parts $\Omega$ :

$$
\frac{1}{2} \frac{d}{d t}\left\|\nabla \rho^{\varepsilon}\right\|_{2, \Omega}^{2}+\int_{\Omega}\left(v^{\varepsilon} \cdot \nabla\right) \rho^{\varepsilon} \Delta \rho^{\varepsilon} d x=0 .
$$

With allowance for the estimate (13) by virtue of the maximum principle it follows that:

$$
\left\|\rho^{\varepsilon}\right\|_{W_{p}^{1,1}(Q)} \leq C<\infty
$$

We have estimating $p^{\varepsilon}$ from the negative norm, as in [7]:

$$
\left\|p^{\varepsilon}\right\| \leq C\left\|\nabla p^{\varepsilon}\right\|<\infty .
$$

It is known that if $v^{\varepsilon}, p^{\varepsilon}$-solution of the following linear Stokes problem:

$$
\begin{gathered}
\mu \Delta v^{\varepsilon}-\nabla p^{\varepsilon}=f ; \\
\varepsilon p^{\varepsilon}+d i v v^{\varepsilon}=0 ; \\
\left.v^{\varepsilon}\right|_{S=0}=0,\left.\frac{\partial \rho^{\varepsilon}}{\partial n}\right|_{S}=0,
\end{gathered}
$$

then provided that $f \in L_{p}(\Omega)$ the following inequality holds:

$$
\left\|v^{\varepsilon}\right\|_{W_{p}^{2} \cap \underset{P^{1}}{\stackrel{0}{W}}}+\left\|p^{\varepsilon}\right\|_{W_{p^{1}}} \leq C\|f\|_{L_{p}} .
$$


We take the function as a function $f$ in problem (17):

$$
f=-\rho^{\varepsilon}\left(\frac{\partial v^{\varepsilon}}{\partial t}+\left(v^{\varepsilon} \cdot \nabla\right) v^{\varepsilon}\right)-\ell \theta^{\varepsilon} \rho^{\varepsilon}-\rho^{\varepsilon} f-\rho^{\varepsilon} \frac{v^{\varepsilon}}{2} \operatorname{div} v^{\varepsilon} .
$$

We estimate the right side by the Cauchy inequality using the maximum principle:

$$
\|f\|_{L_{p}(\Omega)}^{2} \leq C M\left(\left\|v_{t}^{\varepsilon}\right\|^{2}+\int_{\Omega}\left(\left|v^{\varepsilon}\right|^{2}\left|\nabla v^{\varepsilon}\right|^{2}+|f|^{2}+\ell\left|\theta^{\varepsilon}\right|^{2}\right) d x\right) .
$$

We have the following estimate taking into account the inequalities of the embedding and the obtained estimates (13)-(14):

$$
\begin{gathered}
\int_{\Omega}\left|v^{\varepsilon}\right|^{2}\left|\nabla v^{\varepsilon}\right|^{2} d x \leq \max _{\Omega}\left|v^{\varepsilon}\right|^{2} \int_{\Omega}\left|\nabla v^{\varepsilon}\right|^{2} d x \leq\left\|v^{\varepsilon}\right\|_{L_{p}(\Omega)}\left\|v^{\varepsilon}\right\|_{W_{p}^{2}(\Omega) \cap \underset{p^{1}}{W}(\Omega)}\left\|\nabla v^{\varepsilon}\right\|^{2} \leq \\
\leq l e \delta\left\|v^{\varepsilon}\right\|_{W_{p}^{2}(\Omega) \bigcap_{p^{1}}^{(\underset{W}{0}(\Omega)}}^{2}+C_{\delta}\left\|v^{\varepsilon}\right\|_{\substack{W \\
p^{1}}}^{2} .
\end{gathered}
$$

We multiply equation $(7)$ scalarly by $v^{\varepsilon}(t)$ in space $L_{2}(\Omega)$. Then we estimate the integrals in absolute value from above and, applying the inequalities of imbedding theorems, we obtain the following estimate:

$$
\begin{aligned}
& \int_{\Omega}\left|\nabla v^{\varepsilon}\right|\left|v^{\varepsilon}\right|\left|v_{t}^{\varepsilon}\right| d x \leq C\left\|\nabla v^{\varepsilon}\right\|_{L_{p}(\Omega)} \max _{\Omega}\left|v^{\varepsilon}\right|\left\|v_{t}^{\varepsilon}\right\|_{L_{p}(\Omega)} \leq
\end{aligned}
$$

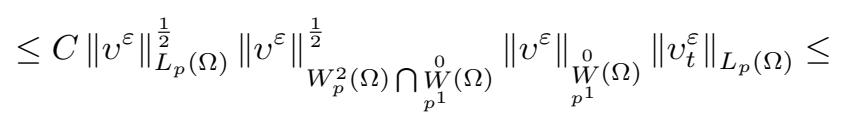

$$
\begin{aligned}
& \leq \delta\left\|v_{t}^{\varepsilon}\right\|_{L_{p}(\Omega)}^{2}+\delta\left\|v^{\varepsilon}\right\|_{W_{p^{2}}(\Omega) \cap \underset{p^{1}}{0}(\Omega)}^{2}+C_{\delta}\left\|v^{\varepsilon}\right\|_{\substack{W \\
p^{1}}}^{2} .
\end{aligned}
$$

We finally get an estimate following the evaluation methodology in the works $[8,9]$ :

$$
\left\|v_{t}^{\varepsilon}\right\|_{L_{p}\left(0, T, L_{p}(\Omega)\right)}+\left\|v^{\varepsilon}\right\|_{L_{p}\left(0, T, W_{p}^{2}(\Omega)\right)}+\left\|\nabla p^{\varepsilon}\right\|_{L_{p}\left(0, T, L_{p}(\Omega)\right)} \leq C<\infty,
$$

where $C$ does not depend on the value of the small $\varepsilon$.

We establish one more estimate of the global time character, the constant in which depends only on the data of the problem. This estimate in the future guarantees compactness in space $L_{2}(Q)$ sequences of approximate solutions, which are built according to the Galerkin method.

Lemma. For any $\delta$, such that the following condition holds $0<\delta<T$, the inequality holds:

$$
\int_{0}^{T-\delta}\left\|v^{\varepsilon}(t+\delta)-v^{\varepsilon}(t)\right\|^{2} d t \leq C \delta^{\frac{1}{2}}
$$

Evidence. We fix $\delta, t$ in such a way that inequality $0 \leq t \leq T-\delta$. Consider equations (7)-(11) on the time interval $\tau \in(t, t+\delta)$. We multiply equation (7) scalarly by $\Phi$ an arbitrary function in space $L_{2}(\Omega)$.

We arrive at the inequality after simple transformations:

$$
\begin{aligned}
& \frac{d}{d \tau}\left(\rho^{\varepsilon} v^{\varepsilon}, \Phi\right)_{L_{2}(\Omega)}=\left(\rho^{\varepsilon}\left(v^{\varepsilon} \cdot \nabla\right) \Phi, v^{\varepsilon}\right)_{L_{2}(\Omega)}+\frac{1}{2}\left(\rho^{\varepsilon} d i v v^{\varepsilon} \cdot v^{\varepsilon}, \Phi\right)_{L_{2}(\Omega)}+ \\
& \quad+\left(\rho^{\varepsilon} f, \Phi\right)_{L_{2}(\Omega)}-\ell\left(\theta^{\varepsilon} \rho^{\varepsilon}, \Phi\right)_{L_{2}(\Omega)}-\mu\left(v^{\varepsilon}, \Phi\right)_{L_{2}(\Omega)}+\left(p^{\varepsilon}, \operatorname{div} \Phi\right)_{L_{2}(\Omega)}
\end{aligned}
$$

where $\Phi=v(t+\delta)-v(t)$.

We integrate this identity with respect to the variable $\tau$ ranging from $t$ before $t+\delta$, and then put $\Phi=v^{\varepsilon}(t+\delta)-v^{\varepsilon}(t)$.

We write expression $\rho^{\varepsilon}(t+\delta) v^{\varepsilon}(t+\delta)-\rho^{\varepsilon}(t) v^{\varepsilon}(t)$ in the following form $\rho^{\varepsilon}(t+\delta)\left(v^{\varepsilon}(t+\delta)-v^{\varepsilon}(t)\right)+$ $+\left(\rho^{\varepsilon}(t+\delta)-\rho^{\varepsilon}(t)\right) v^{\varepsilon}(t)$ and then we find the difference between $\rho^{\varepsilon}(t+\delta)-\rho^{\varepsilon}(t)$ by integrating equation (8) in the range fromtbefore $t+\delta$. The resulting relation is integrated with respect to the variable $t$ from 0 
beforet $+\delta$ and for each term on the right-hand side, we use estimates from $[10,11]$, on the basis of which we derive the estimate of the lemma.

We now turn to the construction of approximate solutions by the Galerkin method.

Let $\left\{\varphi_{j}\right\}_{j=1}^{\infty}$ - orthonormal basis in space $L_{2}(\Omega)$ of $W_{p}^{2}(\Omega) \cap \underset{p^{1}}{\stackrel{0}{W}}(\Omega)$ and the following is true:

$$
\left(\varphi_{j x}, \omega_{x}\right)_{L_{2}(\Omega)}=\lambda_{j}\left(\varphi_{j}, \omega_{j}\right)_{L_{2}(\Omega)} .
$$

Approximate solution $v^{N, \varepsilon}(t)$ looking for in the form:

$$
v^{N, \varepsilon}(t)=\sum_{k=1}^{N} C_{k}^{N}(t) \varphi_{k},
$$

where $C_{k}^{N}(t) \in C^{1}[0, T]$.Density $\rho^{N, \varepsilon}(t)$-there is a classical solution of the problem:

$$
\begin{gathered}
\frac{\partial \rho^{N, \varepsilon}(t)}{\partial t}+\left(v^{N, \varepsilon}(t) \cdot \nabla\right) \rho^{N, \varepsilon}(t)=0 ; \\
\left.\rho^{N, \varepsilon}\right|_{t=0}=\rho_{0}^{M}(x),
\end{gathered}
$$

where $\rho_{0}^{M}(x)$-smooth initial function [12].

Sequence $\rho_{0}^{M}(x), M=1,2 \ldots$ converge to $\rho_{0}(x)$ in the norms $L_{p}(\Omega), W_{p}^{1}(\Omega), \rho_{0}^{M}(x) \in C^{2}(\Omega)$. Pressure $p^{N, \varepsilon}(t)$ - there is a classical solution of the problem:

$$
\begin{gathered}
\operatorname{div} v^{N, \varepsilon}=\varepsilon p^{N, \varepsilon} \\
\int_{\Omega} p^{N, \varepsilon} d x=0 .
\end{gathered}
$$

Temperature $\theta^{N, \varepsilon}(t)$-is defined as the classical solution of the problem:

$$
\begin{gathered}
\rho^{N, \varepsilon}\left(\frac{\partial \theta^{N, \varepsilon}(t)}{\partial t}+\left(v^{N, \varepsilon}(t) \cdot \nabla\right) \theta^{N, \varepsilon}(t)\right)=\operatorname{div}\left(\lambda\left(\theta^{N, \varepsilon}(t)\right) \nabla \theta^{N, \varepsilon}(t)\right)+\mu \sigma^{N, \varepsilon} ; \\
\left.\theta^{N, \varepsilon}\right|_{S=0}=\theta_{0}^{M}(x),\left.\frac{\partial \theta^{N, \varepsilon}(t)}{\partial n}\right|_{S}=0, t \in[0, T],
\end{gathered}
$$

where $\theta_{0}^{M}(x)$ - an initial smooth function satisfying the equation:

$$
\left.\frac{\partial \theta_{0}^{M}(t)}{\partial n}\right|_{S}=0, t \in[0, T]
$$

Functions $C_{k}^{N}(t), \quad k=1,2, \ldots, N$,are determined by a system of ordinary differential equations with coefficients that depend in an operator way on $\rho^{N, \varepsilon}(t), p^{N, \varepsilon}(t)$ :

$$
\begin{gathered}
\left(\rho^{N, \varepsilon}(t)\left(\frac{\partial v^{N, \varepsilon}(t)}{\partial t}+\left(v^{N, \varepsilon}(t) \cdot \nabla\right) v^{N, \varepsilon}(t)+\frac{1}{2} v^{N, \varepsilon}(t) d i v v^{N, \varepsilon}\right)-\mu \Delta v^{N, \varepsilon}(t)+\right. \\
\left.+\nabla p^{N, \varepsilon}(t)-\ell \theta^{N, \varepsilon}(t) \rho^{N, \varepsilon}(t)+\rho^{N, \varepsilon}(t) f, \varphi_{j}\right)_{L_{2}(\Omega)}=0 .
\end{gathered}
$$

We can select subsequences for which we have based on the Schauder principle, using the obtained a priori estimates, from the sequences $\left\{v^{N, \varepsilon}\right\},\left\{\rho^{N, \varepsilon}\right\},\left\{p^{N, \varepsilon}\right\},\left\{\theta^{N, \varepsilon}\right\}$ :

$$
\begin{aligned}
& v^{N, \varepsilon} \rightarrow v^{\varepsilon} \text { weaklyat } L_{p}\left(0, T, W_{p}^{2}(\Omega)\right) ; \\
& \theta^{N, \varepsilon} \rightarrow \theta^{\varepsilon} \text { weaklyat } W_{p}^{2,1}(Q) \\
& \rho^{N, \varepsilon} \rightarrow \rho^{\varepsilon} \text { weaklyat } W_{p}^{1,1}(Q) ; \\
& v^{N, \varepsilon} \rightarrow v^{\varepsilon} \text { strongat } L_{p}\left(0, T, L_{p}(\Omega)\right) \\
& \theta^{N, \varepsilon} \rightarrow \theta^{\varepsilon} \text { strongat } L_{p}\left(0, T, L_{p}(\Omega)\right) ; \\
& v_{t}^{N, \varepsilon} \rightarrow v_{t}^{\varepsilon} \text { weaklyat } L_{p}\left(0, T, L_{p}(\Omega)\right) \\
& p^{N, \varepsilon} \rightarrow p^{\varepsilon} \text { weaklyat } L_{p}\left(0, T, W_{p}^{1}(\Omega)\right) .
\end{aligned}
$$


This completes the proof of Theorem 1 .

The following is valid.

Theorem 2. Suppose that all the conditions of Theorem 1 are satisfied.Then the strong solution of problem

(7)-(12) converges to the strong solution of problem (1)-(6) for $\varepsilon \rightarrow 0$.

Evidence. Because of the a priori estimates obtained earlier, we have:

$v^{\varepsilon} \rightarrow v$ weakly at $L_{p}\left(0, T, W_{p}^{2}(\Omega)\right)$;

$\theta^{\varepsilon} \rightarrow \theta$ weakly at $W_{p}^{2,1}(Q)$

$\rho^{\varepsilon} \rightarrow \rho$ weakly at $W_{p}^{1,1}(Q) ; v^{\varepsilon} \rightarrow v$ strong at $L_{p}\left(0, T, L_{p}(\Omega)\right)$;

$\theta^{\varepsilon} \rightarrow \theta$ strong at $L_{p}\left(0, T, L_{p}(\Omega)\right)$,

$v_{t}^{\varepsilon} \rightarrow v_{t}$ weakly at $L_{p}\left(0, T, L_{p}(\Omega)\right)$;

$p^{\varepsilon} \rightarrow p$ weakly at $L_{p}\left(0, T, W_{p}^{1}(\Omega)\right)$.

Passing to the limit as $\varepsilon \rightarrow 0$ in the corresponding identities, we establish that the limit functions $v, p, \rho, \theta-$ there is a strong solution of problem (1)-(6).

Theorem 2 is proved.

\section{References}

1 Thiele A. A weighted upwind finite element method forolving the stationary Navier-Stokes equations / A.Thiele, L.Tobiska // Wiss. Tech. Univ. -Magdeburg. - 1989. - Vol. 33. - No. 6. - P. 13-20.

2 Prosnak W.J. On a new method for numerical solution of the Navier-Stokes equations / W.J. Prosnak, Z.J. Koma // Acta Mech. - 1991. - Vol. 89. - No. 1. - P. 45-63.

3 Sciubba E.A. Variational derivation of the Navier-Stokes equationsbased on the exergy destruction of the flow / E.A. Sciubba // J. Math. And Phys. Sci. - 1991. - Vol. 25. - No. 1. - P. 61-68.

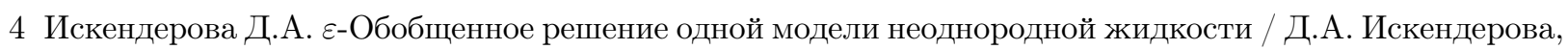
Б.Ж. Сагиндиков // Вестник КазНУ. Сер. Математика, Информатика. - 1998. - № 11. - С. 53-65.

5 Кажикенова С.Ш. Теоремы о существовании решения одной стационарной модели несжимаемого расплава / С.Ш. Кажикенова // Труды университета. - 2008. - № 3(32). - С. 28-30.

6 Кажихов А.В. О корректности краевых задач в одной диффузионной модели неоднородной жидкости / А.В. Кажихов, Ш.С. Смагулов // ДАН СССР. - 1977. - Т. 234. - № 2. - С. 330-332.

7 Куттыкожаева Ш.Н. $\varepsilon$-Аппроксимация модели уравнения свободных конвекций с учетом переменных функций тока и вихря скоростей / Ш.Н. Куттыкожаева, С.Ш. Кажикенова // Вестник КазНУ. Сер. Математика, Информатика. - 2002. - № 2(30). - С. 93-95.

8 Abylkairov U.U. On the $\varepsilon$-approximation for the Modified equations of the heat convection / U.U. Abylkairov, S.T. Mukhametzhanov, Kh.Khompysh // Universal Jornal of Mathematics and Mathematical Sciences. - India: Pushpa Publishing Allahabad, 2004. - Vol. 5. - No. 1. - P. 37-51.

9 Meirmanov A. Mathematical model of a liquid filtration from reservoirs / A. Meirmanov, S. Mukhametzhanov // Electronic // Universal Jornal of Dierential Equations. - Vol. 2014(2014). - No. 49. - P. 1-13.

10 Антонцев С.Н. Краевые задачи механики неоднородных жидкостей / С.Н. Антонцев, А.В. Кажихов, В.Н. Монахов. - Новосибирск: Наука, 1938. - С. 318.

11 Камаубаев К.С. Об одном приближенном методе решения уравнений Навье-Стокса в естественных переменных / К.С. Камаубаев, Ш.С. Смагулов, Н.М. Темирбеков // Доклады Академии наук РК. - 1999. - № 5. - С. 10-15.

12 Кажикенова С.Ш. Аппроксимация стационарной модели неоднородной несжимаемой жидкости / С.Ш. Кажикенова // Вестн. Кузбас. гос. техн. ун-та. - Кемерово, 2010. - № 6. - С. 113-116. 
С.Ш. Кажикенова, М.И. Рамазанов, А.А. Хайркулова, Г.С. Шаихова

\title{
Энергияны диссипациялауға арналған жолмен біртекті емес балқыламалардың температуралық моделін $\varepsilon$-жуықтауы
}

\begin{abstract}
Үлкен сандармен тәжірибе және жуықтау есептеулер жасауға байланысты Навье-Стокс теңдеуі туралы жинақталған мәліметтерден табиғаттағы нақты құбылыстардың нақты балқымалар жүйесі мен тұтқыр балқымалардың математикалық моделі арасындағы сәйкестікті байқауға мүмкіндік береді. Бұл үшін көптеген себептер бар. Олардың бірі - сызықты емес Навье-Стокс теңдеуі. Сызықты емес теңдеулер үшін стационарлы емес есептерді қанағаттандыратын шешімдері $t \geq 0$ интервалында барлығында болмауы мүмкін екені белгілі. Соңғы уақыт аралығында шексіздікке ұмтылуы мүмкін немесе құлдырайды, яғни тұрақтылығын жоғалтады, теңдеуді қанағаттандырмайды және тармақтала бастайды. Егер осы шешім $t \geq 0$ болғанда, онда ол сыртқы әсер мен шекаралық шарттарды түрақтандыру кезінде стационарлы есептің шешіміне ұмтылмауы математикалық тұрғыда дәлелденді. Алынған стационарлы емес есептің шешімі тегіс бастапқы тәртіпте және тегіс сыртқы әсерлерде уақыт өте тұрақтылығын азайта береді. Содан кейін тұрақсыз немесе турбулентті режимге көшеді. Шешімнің бір немесе бірнеше тармағын нақты іске асыру Навье-Стокс теңдеуінде ескерілмеген кез келген себептерге байланысты. Ұсынылған мақалада жақсы жуықтау арқылы сандық схема құрылды. $\varepsilon$-Жуықтауы арқылы дифференциалдық теңдеулердің бастапқы жүйелері регуляризацияланды. Сандық және аналитикалық түрде сығылмайтын тұтқыр ағым үшін шекаралық есептердің дұрыстығын зерттеуді қамтамасыз ететін Галеркин әдісі енгізілді. Әлсіз жуыктауы бар Навье-Стокс теңдеуі үшін бөлу схемасы құрылды. Гидродинамиканың сызықты емес теңдеуін Коши-Ковалевский типті теңдеулер жүйесіне келтіруге болатындай сығылмайтын балқыманың стационарлы және стационарлы емес моделдерін жуықтау қолданылды.
\end{abstract}

Kiлm сөздер: энергияны диссипациялау, жүйені жақындату, Коши теңсіздігі, Галеркин әдісі, априорлық бағалау.

\section{С.Ш. Кажикенова, М.И. Рамазанов, А.А. Хайркулова, Г.С. Шаихова \\ $\varepsilon$-Аппроксимация температурной модели неоднородных расплавов с учетом диссипации энергии}

\begin{abstract}
Накопленные факты и сведения об уравнениях Навье-Стокса, наряду с большим числом экспериментов и приближенных расчетов, позволили выявить некоторые несоответствия между математической моделью вязкого расплава и реальными явлениями в природе реальных расплавленных систем. И этому есть много причин. Одна из них - нелинейность уравнений Навье-Стокса. А для нелинейных уравнений известно, что в нестационарных задачах удовлетворяющее им решение может существовать не на всем интервале $t \geq 0$. За конечный промежуток времени оно может либо уйти в бесконечность, либо рассыпаться, т.е. потерять регулярность и перестать удовлетворять уравнениям и начать ветвиться. Математически доказано, что если же это решение существует при $t \geq 0$, то оно может не стремиться к решению стационарной задачи при стабилизации краевых условий и внешних воздействий. Полученные решения нестационарной задачи даже при гладком начальном режиме и гладких внешних воздействиях могут со временем становиться менее регулярными, а затем вообще перейти в нерегулярные или турбулентные режимы. Фактическая реализация той или иной ветви решения зависит от посторонних причин, неучтенных в уравнениях Навье-Стокса. В предлагаемой статье построена численная схема, обладающая хорошей сходимостью. Построена регуляризация исходных систем дифференциальных уравнений путем $\varepsilon$-аппроксимации. Реализован метод Галеркина, обеспечивающий изучение корректности краевых задач для несжимаемого вязкого потока как численно, так и аналитически. Построена схема расщепления для уравнений Навье-Стокса со слабой аппроксимацией. Построена аппроксимация стационарной и нестационарной моделей несжимаемого расплава, что приводит нелинейные уравнения гидродинамики к системе уравнений типа Коши-Ковалевской.
\end{abstract}

Ключевые слова: диссипация энергии, аппроксимация системы, неравенство Коши, метод Галеркина, априорные оценки. 


\section{References}

1 Thiele, A., \& Tobiska, L. (1989). A weighted upwind finite element method forolving the stationary Navier-Stokes equations. Wiss. Tech. Univ. -Magdeburg, Vol. 33, 6, 13-20.

2 Prosnak, W.J., \& Koma, Z.J. (1991). On a new method for numerical solution of the Navier-Stokes equations. Acta Mech., Vol. 89, 1, 45-63.

3 Sciubba, E.A. (1991). Variational derivation of the Navier-Stokes equationsbased on the exergy destruction of the flow. J. Math. And Phys. Sci., Vol. 25, 1, 61-68.

4 Iskenderova, D.A., \& Sagindikov, B.Zh. (1998). $\varepsilon$-Obobshchennoe reshenie odnoi modeli neodnorodnoi zhidkosti [ $\varepsilon$-Generalized solution of the model of inhomogeneous liquids]. Vestnik KazNU. Seriia Matematika, informatika - Bulletin KazNU. Series Mathematics, Informatics, 11, 53-65 [in Russian].

5 Kazhikenova, S.Sh. (2008). Teoremy o sushchestvovanii resheniia odnoi statsionarnoi modeli neszhimaemoho rasplava [Theorems on the existence of a solution of a stationary model of an incompressible melt]. Trudy universiteta - Proceedings of the University, 3(32), 28-30 [in Russian].

6 Kazhikhov, A.V., \& Smagulov, Sh.S. (1977). O korrektnosti kraevykh zadach v odnoi diffuzionnoi modeli neodnorodnoi zhidkosti [On the well-posedness of boundary value problems in a diffusion model of an inhomogeneous fluid]. Doklady Akademii nauk SSSR - Reports of the Academy of Sciences SSSR, Vol. 234, 2, 330-332 [in Russian].

7 Kuttykozhava, Sh.N., \& Kazhikenova, S.Sh. (2002). $\varepsilon$-Approksimatsiia modeli uravneniia svobodnykh konvektsii s uchetom peremennykh funktsii toka i vikhria skorostei [ $\varepsilon$-Approximation of the model of the free convection equation with allowance for the variables of the current function and the velocity vortex]. Vestnik KazNU. Seriia Matematika, informatika - Bulletin KazNU. Series Mathematics, Informatics, 2(30), 93-95 [in Russian].

8 Abylkairov, U.U., Mukhametzhanov, S.T., \& Khompysh, Kh. (2004). On the $\varepsilon$-approximation for the Modified equations of the heat convection. Universal Jornal of Mathematics and Mathematical Sciences, Vol. 5, 1, 37-51. India: Pushpa Publishing Allahabad.

9 Meirmanov, A., \& Mukhametzhanov, S. (2014). Mathematical model of a liquid filtration from reservoirs. Electronic. Universal Jornal of Dierential Equations, Vol. 2014, 49, 1-13.

10 Antontsev, S.N., Kazhikhov, A.V., \& Monakhov, V.N. (1938). Kraevye zadachi mekhaniki neodnorodnykh zhidkostei [Boundary value problems for the mechanics of inhomogeneous liquids]. Novosibirsk: Nauka [in Russian].

11 Kalumbaev, K.S., Smagulov, Sh.S., \& Temirbekov, N.M. (1999). Ob odnom priblizhennom metode resheniia uravnenii Nave-Stoksa $\mathrm{v}$ estestvennykh peremennykh [On an approximate method for solving the Navier-Stokes equations in natural variables]. Doklady Akademii nauk RK-Reports of the Academy of Sciences RK, 5, 10-15 [in Russian].

12 Kazhikenova, S.Sh. (2010). Approksimatsiia statsionarnoi modeli neodnorodnoi neszhimaemoi zhidkosti [Approximation of a stationary model of an inhomogeneous incompressible fluid]. Vestnik Kuzbasskoho hosudarstvennoho tekhnicheskoho universiteta - Bulletin of the Kuzbass State Technical University, 6, 113-116. Kemerovo [in Russian]. 